\title{
Topónimos documentados en el Libro de Apeos de Mansilla Mayor (León, 1746) / Place names documented in the Libro de Apeos of Mansilla Mayor (León, 1746)
}

\author{
TERESA LLAMAZARES PRIETO \\ UNIVERSIDAD DE LEÓN \\ ORCID 0000-0002-2188-1412
}

\begin{abstract}
RESUME: El Llibru d'Apeos de Mansilla Mayor ye un documentu del sieglu XVIII, manuscritu nun tomu y curiáu na ilesia de la mesma localidá, onde se rexistren les llendes de toles posesiones de los habitantes d'aquel entós, incluyíes les de la mesma ilesia. Mansilla Mayor ye un conceyu de la provincia de Lleón, asitiáu na fastera o comarca conocida anguaño como Esla-Campos. Ente'l so patrimoniu arqueolóxico y monumental atópase l'asentamientu de la ciudá ástur y romana de Lancia y el monesteriu cisterciense de Santa María de Sandoval (sieglu XII). Llingüísticamente falando, la zona asítiase nel asturianolleonés central. Esti trabayu arrexunta tolos topónimos citaos nesi documentu y constata aquellos qu'inda güei son conocíos polos sos vecinos y que suponen un 35 ó $40 \%$ de los documentados nesi manuscritu. Del analís del documentu constátase la presencia de formes toponímiques tradicionales (como Llama, Castro, prefixu so- y sobre-, abondancial en -al, caltenimientu del grupu - $m b$-, ente otres) lo que dexa algamar conclusiones rellacionaes con usos llingüísticos del antiguu dominiu ástur. Nesti sentíu, y en rellación al vocabulariu, ye interesante atopar documentaes voces con un usu anguaño desapaecíu dafechu o que ta en francu retrocesu, como les palabres ejido, ferreñal o ferrenal o dos abondanciales fitotoponímicos como olmar y ponjal. La concentración parcelaria de la zona y l'amenorgamientu esmolecedor de la población del conceyu faen urxente la coyida y documentación d'esi patrimoniu toponímicu en peligru de desapaición.
\end{abstract}

Pallabres clave: llibros d'apeos; toponimia; sieglu XVIII, asturianolleonés central.

AbSTRACT: The «Libro de Apeos» (i.e., Land Registry file) of Mansilla Mayor is an $18^{\text {th }}$ century document, handwritten in one volume and kept in the town's church, in which the boundary lines of all the land of the inhabitants of that time, including those of the church itself, are recorded. Mansilla Mayor is a town in the province of León, located in the Esla-Campos Leonese region. Among its archaeological and monumental heritage is the settlement of the Asturian and Roman city of Lancia and the Cistercian monastery of Santa María de Sandoval $\left(12^{\text {th }}\right.$ century). Linguistically speaking, the area is located within the central Asturian-Leonese dialect. This paper compiles all the place-names cited in that document and verifies those that are still known by the town's residents, which are reduced to 35 or $40 \%$ of all the place-names documented in that manuscript. The analysis of the document demonstrates the existence of traditional toponymic forms (such as Llama, Castro, prefix so- and sobre-, 
abundant in $-a l$, keeping the group $-m b$-, among others), which allows us to draw conclusions related to linguistic uses in the Old Leonese territory. In this sense, and in relation to vocabulary, it is interesting to find documented voices whose use has currently disappeared completely or is in frank retreat, such as the words ejido, ferreñal or ferrenal or two abundant phytoponimics such as olmar and ponjal. The combination of properties undergone in the area as well as the alarming decrease in the population of the municipality make the collection and documentation of this toponymic heritage that is in danger of disappearing urgent.

Keywords: Land Registry Document; toponymy; $18^{\text {th }}$ century, central Asturian-Leonese.

\section{INTRODUCCIÓN}

Simplificando mucho, se podría afirmar que la investigación toponímica persigue dos grandes objetivos: recoger topónimos y estudiarlos para desentrañar su significado; clasificarlos y documentarlos. En esa recogida se intuyen antiguas formas de vida, culturas diversas y pueblos que ya han desaparecido, pero que dejan, entre otros testimonios, los nombres de lugar. Y estas etiquetas colocadas a lo largo y ancho de la geografía, son como indicadores para otras ramas del quehacer humano. Así, un historiador puede conocer lo que Unamuno llamó intra-historia, la vida secular de villas y aldeas, con sus granjas, sus cultivos, sus molinos, sus santuarios locales, sus costumbres, sus consejas (Lapesa 1969: 239-251) y, por supuesto, la gran historia, ya que se podría afirmar que cada uno de los episodios importantes, léase invasiones de otros pueblos, romanización, la Reconquista y repoblación, etc., está reflejado en multitud de nombres de lugar.

Pero no es necesario buscar razones externas que justifiquen los estudios toponímicos porque coincidimos con la opinión expresada por González y Fernández-Valles (1959: 8), y recogida por J. Miranda Pérez-Seoane (1985: 7), de que la solución a muchos problemas toponímicos generales está en el estudio detallado de la toponimia integral de pequeñas comarcas, ya que en ellas los topónimos permanecen referidos a sus primitivos objetos o, en todo caso, resultarían fáciles de identificar mediante un minucioso y circunstanciado conocimiento local. Y este es el caso de la zona que nos ocupa.

De las inquietudes de una asociación cultural de Mansilla Mayor surgió el encargo de estudiar la toponimia de esta localidad, a la que me unen estrechos y profundos lazos familiares, encargo apremiante porque la zona sufrió una concentración parcelaria entre 1984 y 1994 que ha supuesto una transformación total del paisaje, con desforestaciones masivas, allanamientos y desecación de terrenos, trazado de nuevos caminos y desaparición de los antiguos, construcción de canales de riego, etc. Urgía, pues, la recogida de la toponimia de boca de aquellas personas que todavía recordaban los nombres de los lugares tal y como estaban antes de la concentración parcelaria, lo que 
facilitaría probablemente su interpretación.

En esa recogida hemos seguido un método riguroso y científico, esbozado ya por Corominas (1972: I, 157; 160): en primer lugar, tener un conocimiento de primera mano de la pronunciación popular del nombre (por eso hemos buscado a informantes entre las personas de más edad del pueblo), buscar variantes de esos nombres en zonas próximas lingüísticamente hablando y rastrear la documentación para encontrar esos topónimos documentados. Una fase inicial de este proyecto es enumerar los topónimos que hemos encontrado documentados en el Libro de Apeos de Mansilla Mayor, documento que se encuentra custodiado en la iglesia de este pueblo. La finalidad de este trabajo no es, por tanto, el estudio detallado de la toponimia del pueblo (excedería los límites destinados a los artículos de esta revista) sino el que acabamos de decir: rastrear la información toponímica consignada en un documento inédito del año 1746.

Mansilla Mayor es un municipio de la provincia de León que incluye las localidades de Mansilla Mayor, Nogales, Villamoros de Mansilla y Villaverde de Sandoval. Situado en la comarca leonesa Esla-Campos, su territorio se asienta en la llanura aluvial de los ríos Esla y Porma, terreno fértil origen de una economía agropecuaria. No es un municipio grande ni en extensión (no llega a $15 \mathrm{~km}^{2}$ ) ni en población, puesto que, según datos del INE, en la actualidad, ronda los 350 habitantes, aunque alcanzó los 1.000 en los años 30 del s. XX.

Entre su patrimonio arqueológico y monumental se encuentra el asentamiento de la ciudad ástur y romana de Lancia, el monasterio cisterciense de Santa María de Sandoval (siglo XII) y la iglesia parroquial de San Miguel (Mansilla Mayor) con dos importantes artesonados, uno mudéjar (sobre la nave central) y otro barroco (sobre el presbiterio). Es un municipio recorrido por El Camino de Santiago Francés y por la Cañada Real Leonesa Occidental.

Esta zona se inscribe, según Menéndez Pidal (2018: 53), en el dialecto central del viejo dominio ástur, con $f$ - (< lat. F-) inicial conservada. No obstante, podemos afirmar que el habla local es castellano con algunos términos asturianoleoneses. La misma explicación que Morala (1984: 21) da para una zona cercana del Esla, encabezada por Palanquinos, vale para la nuestra: una progresiva castellanización ejercida ya desde la época medieval, intensificada en el siglo pasado y en lo que llevamos de este que hace que el asturianoleonés se convierta en un sustrato de la lengua más extendida, el castellano.

Aunque la castellanización estuviera ya muy avanzada en la época en que se data el documento (siglo XVIII), la importancia del mismo radica en que refleja la situación de la lengua del viejo dominio en esa época, especialmente en el campo del léxico. Esa fluctuación entre castellano y asturianoleonés se observa en la conservación unas veces sí y otras no del grupo - $m b$ - o de la $f$ inicial (lombo, pero palomar; ferreñal y erreñal). Pero son numerosos los ejemplos de castellanización como el sufijo -illo, -illa (< -ĚLLUM, -ĚLLAM) 
nunca -iello, -iella (El Pradillo, La Pontonzilla), la no palatalización de L- (Las Lagunas, Los Lombos, Los Lavaderos) o la presencia del fonema velar fricativo frente al fricativo prepalatal sordo (La Requejada y no *La Requexada).

\section{El LibRo DE APEOS DE MANSILla MAYOR}

Precisamente en esa iglesia de San Miguel hemos localizado el Libro de Apeos, Aniversarios, Fundaciones, Cofradías de Mansilla Mayor. Según el Diccionario de la Real Academia Española, apeo es «un documento jurídico que acredita el deslinde y la demarcación» y apear es «reconocer, señalar o deslindar una o varias fincas, y especialmente las que están sujetas a determinado censo, foro u otro derecho real». En este sentido, un Libro de Apeos se convierte en una fuente histórica de gran interés como pone de manifiesto Ponce Molina (1977: 289) cuando alude a los Libros de Apeos y Repartimientos, encargados por Felipe II para la regulación y constancia, a efectos legales y tributarios, del reparto de los bienes que pertenecieron a moriscos o a cristianos viejos en el antiguo reino granadino. Otro ejemplo podría ser El Libro del Prior o apeo del Concejo de Oviedo, del s. XV, donde se consignan las lindes de todas las posesiones del Cabildo de la Catedral de Oviedo (García-Sampedro 2009). Libro de apeo o simplemente apeo es la denominación que recibieron en la España de la Edad Moderna los documentos que combinaban registros catastrales, fueros, derechos y privilegios, sucesos históricos reseñables y jurisdicciones de un lugar, entidad política o religiosa. Por similitud de contenidos, la expresión libro de apeo es sinónima a la de libro becerro, circunscrito casi exclusivamente a la Edad Media.

El Libro de Apeos, Aniversarios, Fundaciones, Cofradías de Mansilla Mayor (en adelante LAA-1) es un documento del año 1746. Tiene tapas de piel, mide 30,5 x $21 \mathrm{~cm}$ y está compuesto de 127 páginas: cada página tiene cara a o recto, y cara b o vuelto (nos referiremos a ellas como $r$ y $v$ respectivamente), es decir, el doble de caras que de páginas. En esas páginas se recoge desde el procedimiento e incidencias del apeo hasta la capacidad y calidad de las tierras o prados, aludiendo incluso a la naturaleza de sus cultivos. Y lo que más nos interesa en este caso, al inventariar minuciosamente los bienes que pertenecían a la iglesia del pueblo o a los habitantes, aparecen los nombres de esas tierras y prados. La estructura del mismo es la siguiente:

- Las páginas 1-14r contienen las diligencias que don Marcos Gutiérrez, juez, debe hacer constar para inscribir en el libro las propiedades que pertenecen a la iglesia o a alguno de los vecinos. Así hay citaciones, requerimientos, notificaciones... para instituir el protocolo de apeos. Se hace referencia a «figuras» que hacían falta para darle legalidad como apeadores, provisores, notarios y se hacía constar el aviso a todos los vecinos a los que competía el 
deslinde de los bienes. Vecinos no solo de Mansilla Mayor sino de otras poblaciones cercanas que pudieran tener propiedades en el territorio del municipio, como Villaverde de Sandoval, Valle de Mansilla... En estas páginas no hay más topónimos que los nombres de los pueblos y encontramos ya un dato interesante: aparece citado varias veces el lugar de Santovenia con la misma entidad o categoría que Villaverde o Mansilla Mayor, lo que atestigua que, lo que hoy en día es un despoblado, en el año 1746 era un pueblo más del municipio. Compárese cómo se citan de la misma manera en página 9r y 10r dos lugares: Santovenia (ya no existe como pueblo) y Villamoros (sigue existiendo): «En el lugar de Santo benia a dos días del mes de noviembre de mil setezientos y quarenta y seis años...»; «En el lugar de villa moros...». El lugar de Santovenia aparece citado luego varias veces cuando se delimitan las posesiones. Por ejemplo, en página $47 \mathrm{r}$ se dice $« \ldots$ con el camino que ba de el lugar de santo benia para el molino»; en 48v, «que linda... azia las partes de arriba y poniente con el camino que ba de Santo benia para Mataganado». En la página $104 \mathrm{r}$ se puede leer: «... otra tierra que esta en el termino y casco del lugar de santobenia que ahora es Guerto...». También aparece el nombre de algún vecino de Santovenia, como en página 106r: «Aniversario Que goza Antonio Prieto vez ${ }^{\circ}$ del lug de Sto benia».

- Páginas $14 \mathrm{v}-115 \mathrm{v}$. Inventario de las tierras de la Iglesia de Mansilla Mayor y de otros propietarios. Para ello se habla de una tierra o prado que sitúan en algún paraje («... a do llaman... .») de Mansilla Mayor o de alguno de los pueblos que lo circundan, se hace referencia a su producción y/o calidad y terminan enumerando sus límites. Por ejemplo:

«... una tierra a do llaman Laguna Salgada que aze de senbradura dos eminas trigal que linda azia las partes del oriente y arriba con tierras de las Monjas de gradefes y abajo con lindero de conzejo y al piniente con lindero de conzejo y la atraviesa la madriz grande de consejo»

- Páginas 116r-119r. Inventario de los bienes y «alajas» que tiene la Iglesia. Se enumeran cosas como «un copón de plata dorado por dentro...», un veril de plata, tres chrismeras, una cruz, vinajeras, casullas...

- Páginas 119r y120r. Diligencias y autos para atender a reclamaciones por si alguien no estuviera conforme con el apeo.

- Páginas 121r y122r. Tasación, es decir, lo que cobraron por hacer el apeo.

- Páginas 122v-123v. Autos y compulsas finales.

- Las páginas 124, 125 y 126 están en blanco y en la página 127 (caras r y v) hay una especie de índice cuyo contenido se transcribe a continuación: 


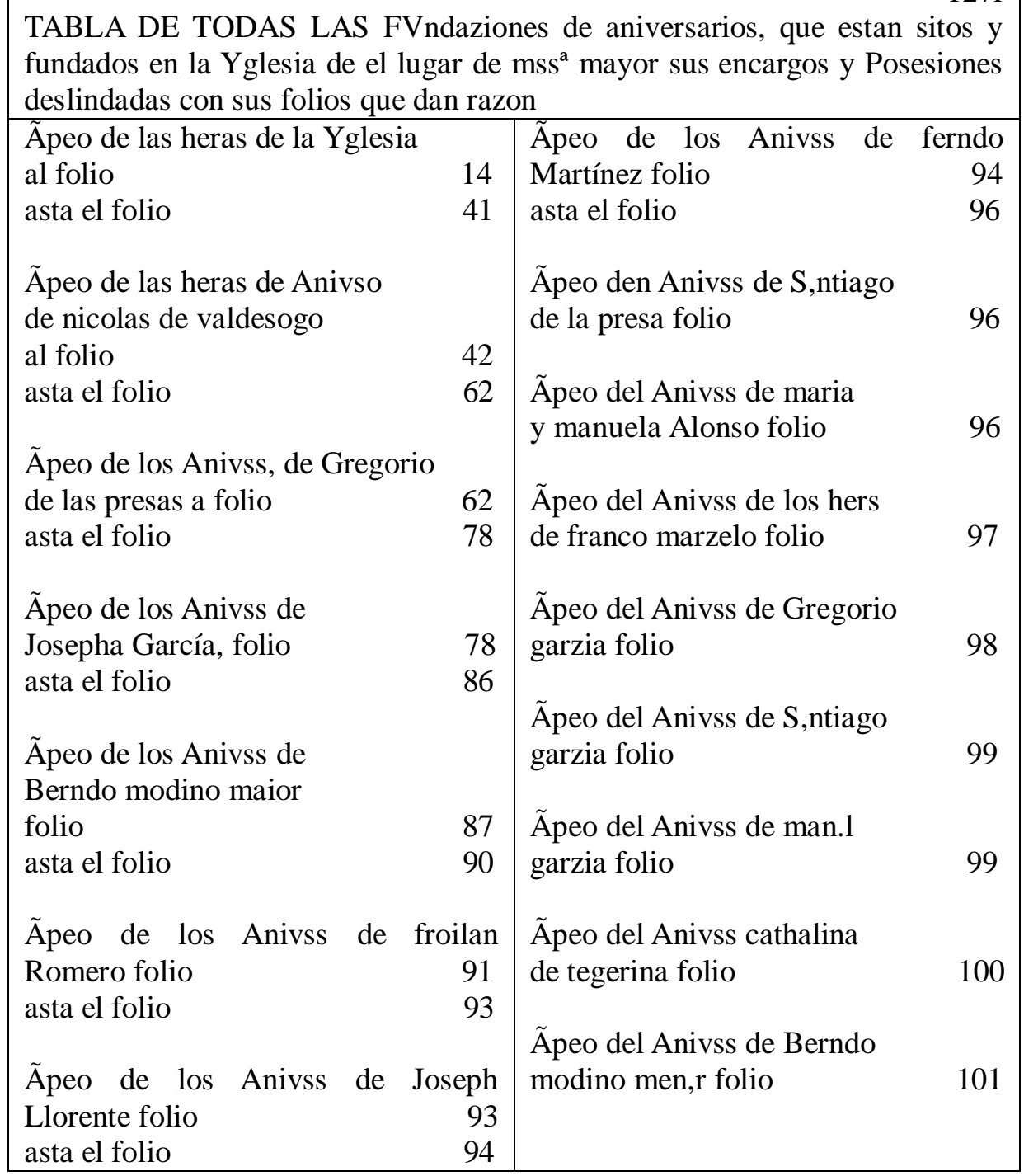

Imagen 1a. Índice del LAA-1. Página 127r 


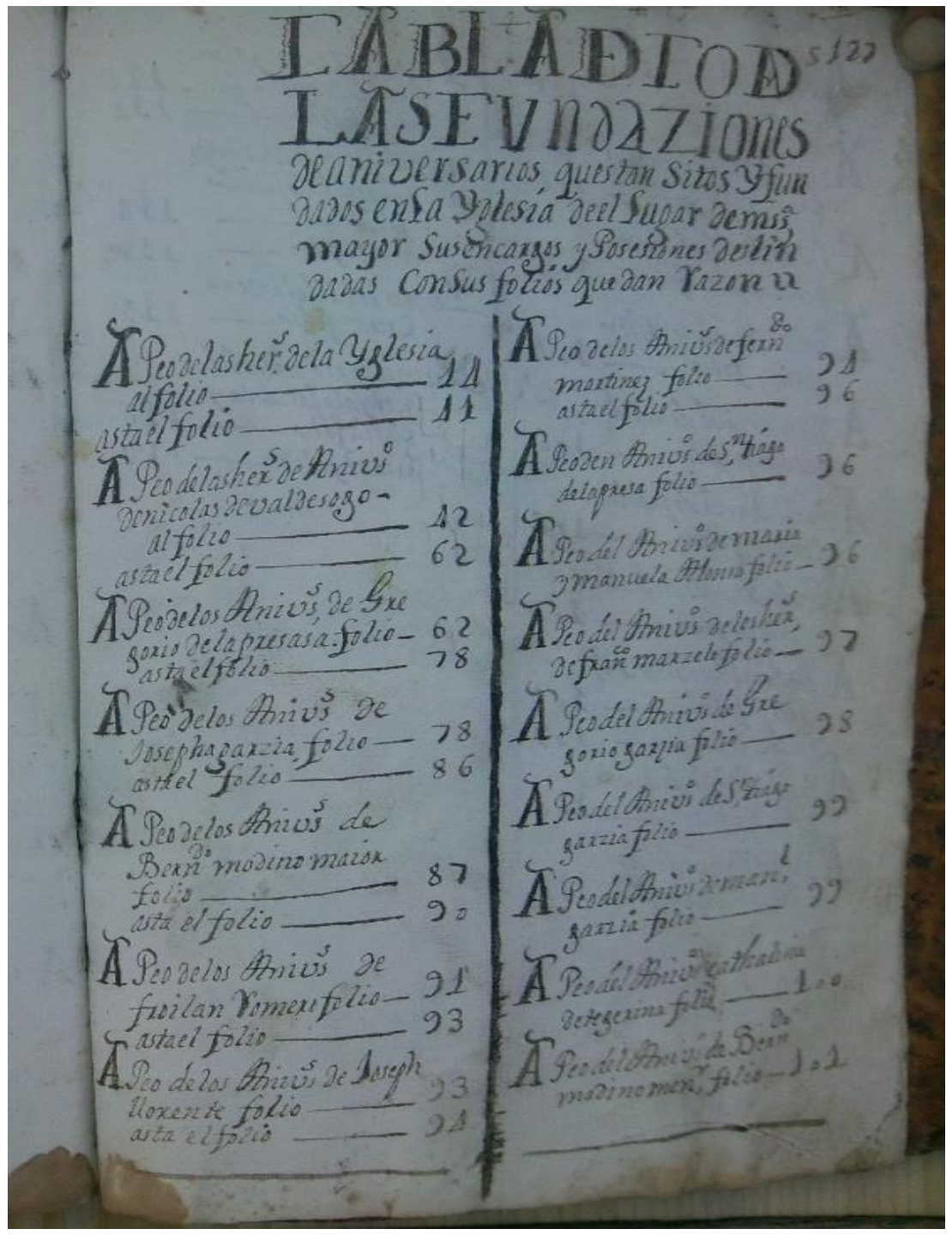

Imagen 1b. Índice del LAA-1. Página 127r 


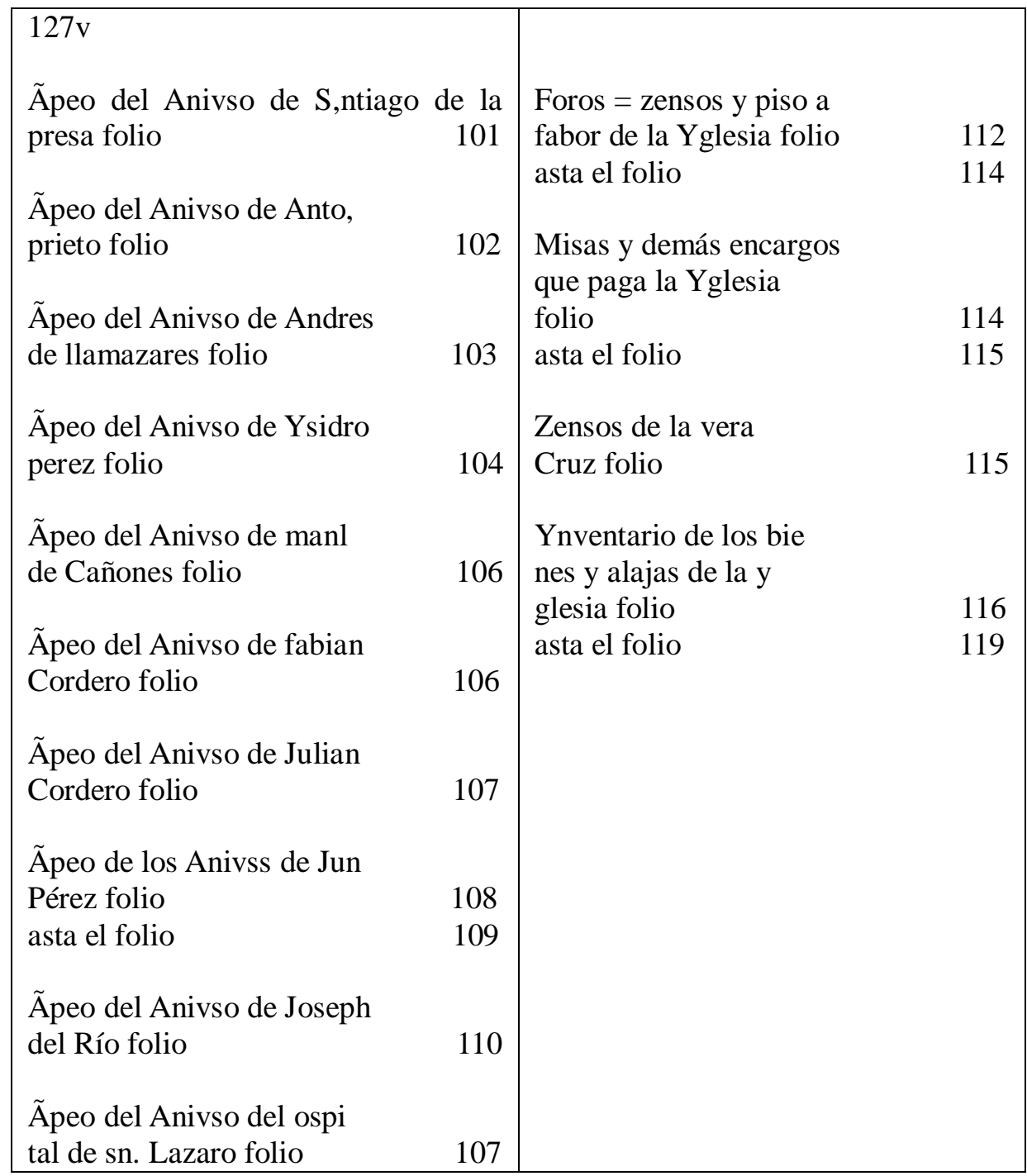

Imagen 2a. Índice del LAA-1. Página 127v 


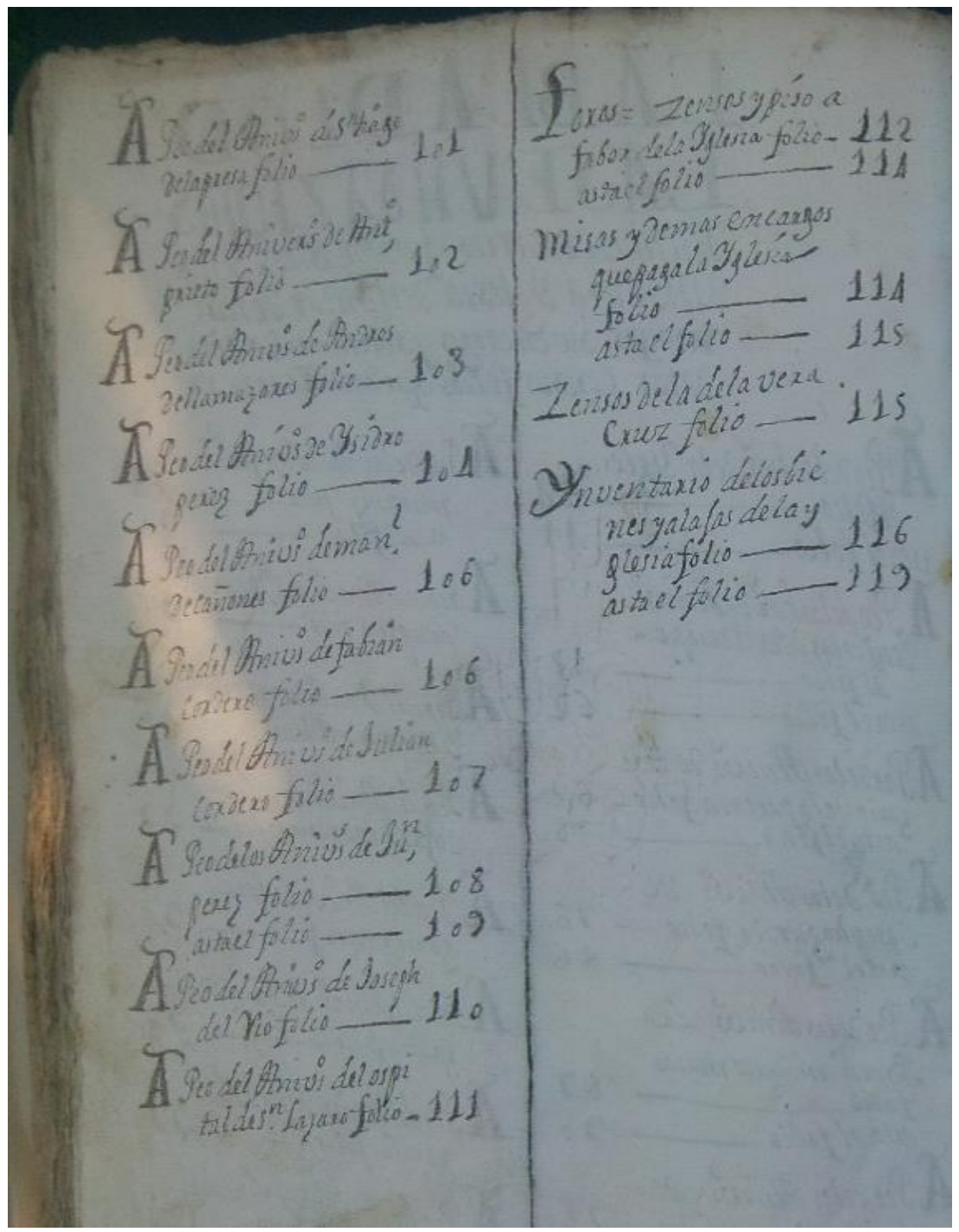

Imagen 2b. Índice del LAA-1. Página 127v 


\section{Topónimos documentados en el Libro de Apeos de Mansilla MAYOR}

Siguiendo ese índice, las páginas más interesantes para nuestra finalidad son las comprendidas entre la $14 \mathrm{v}$ y la $115 \mathrm{v}$ dado que al deslindar las propiedades de la iglesia o de los individuos, aparecen los topónimos. Para exponer esos nombres de lugar que aparecen allí documentados utilizaremos el orden alfabético de la primera palabra del topónimo que no sea el artículo (por ejemplo, El Condado estará en la «c»). Aunque respetaremos la ortografía que hemos encontrado en el Libro de Apeos, incorporaremos el topónimo documentado en la letra que le corresponde según la grafía normativa actual, de manera que si aparece varrio estará en la «b», los zentenales estará en la «c»o el berjel estará colocado en la «V». Destacaremos cada topónimo (con sus variantes) en letra cursiva y figurará entre paréntesis en qué página o páginas del LAA-1 aparece, especificando si está en la cara r o v de la página.

La mayoría de los topónimos se refieren a lugares de Mansilla Mayor, pero tanto la iglesia como los vecinos de este pueblo tenían también propiedades en pueblos vecinos, que recogemos al final de este corpus de topónimos documentados de Mansilla Mayor.

\section{Allende el Camino}

«... una tierra que esta en el termino deste otro lugar a do llaman allende el camino ... que linda ... al poniente con el camino Real.» (20v).

Aparece de la misma manera en 21r y 59r y muy probablemente fuera el mismo lugar que el que consignamos a continuación. El término allén en asturiano significa 'de la parte de allá' y se encuentran variantes como allende, allendi o alliende (DGLA). Topónimos semejantes se encuentran en Asturias, como La Llende o Allande (García Arias 2005).

\section{Allende el Camino Real}

«... a do llaman allende el camino Real ...» (81r).

Bariona, La

«... a do llaman los cogullares... que linda... y azia abajo con tierra que llaman de la bariona» (50r).

Barreros, Los

(Ver Prado de los Barreros).

Barriales, Los

«... a do llaman los barriales...» $(21 \mathrm{v}, 51 \mathrm{v}, 69 \mathrm{v}, 74 \mathrm{v}, 80 \mathrm{v}, 81 \mathrm{v}) ; \ll \ldots$ a do

llaman los brarriales... que linda azia la parte de abajo con el camino de 
los barriales...». (70r); «bariales» (aparece al margen en 51r); «... a do llaman los varriales (de villa moros)... que linda... y arriba con el camino Real» (55r); «... a do llaman los varriales... que linda... y azia la parte de arriba con la madriz de conzejo» (82r).

Conocido hoy en día como Los Barriales. García Arias (2005) afirma que topónimos asturianos como El Barrial, Barriéu podrían ser abundativos si se considera barriu variante de barru.

Barrio de Arriba, El

«... a do llaman el varrio de arriba...» (113r).

Bragada, $L a$

«... otra tierra que esta en el termino deste lugar de Mansilla Mayor a do llaman la braga que aze una emina de senbradura trigal que linda...».

Pero en el margen pone «Otra a la bragada» (16r), forma que coincide con el topónimo actual (La Bragada), de donde se deduce que la forma la braga es un error de escritura.

Calzada, La

«... a do llaman la calzada ... que linda al oriente con el camino Real...» (70v).

La voz calzada, 'camín' o 'carretera', 'camino empedrado', aparece tanto en el DALLA como en el DGLA y en topónimos asturianos como Calzada, La Calzá, Les Calzaes o El Calzáu.

Camino de los Barriales, El (70r)

(Ver Barriales).

Camino de la Carrera, El

«... a do llaman el castro... que linda azia la parte de arriba con el camino de la carrera y al oriente con lindero de conzejo que tiene una poza» (56r).

Hoy en día se conoce con el mismo nombre: El Camino la Carrera.

Camino del Castro, El

«... a do llaman el camino que ba para el Castro ... que linda... y azia el oriente y arriba con la rraya que dibide los términos de Mansilla Mayor y villa sabariego» (65r).

Entre las muchas acepciones que el DGLA recoge para castru, la número 7 es 'castillo, campamento, pueblo fortificado', 'real o sitio donde está acampado y fortificado un ejército'. Conocido es que los romanos denominaron CASTRUM a aquellos poblados de aspecto fortificado que se iban encontrando en su conquista del territorio. De ahí la abundancia de topónimos con esta base que recoge García Arias (2005). 
Camino de la Era, El

«... otra tierra que esta en el termino de santo benia y mansilla Mayor a do llaman la era ... que linda... y al poniente con el camino de la era» (54r).

Camino de la Era el Barrio, El

«... que linda azia el oriente con tierras digo con el camino de la hera del varrio...» (16v).

Camino de las Duernas, El

«... otra tierra que esta en el termino deste otro lugar a do llaman las duernas que aze una fanega de senbradura trigal que linda... y de la parte de abajo con el camino que llaman de las duernas» (19r); «... a do llaman los zentenales... que linda... azia la parte de arriba con el camino que llaman de las duernas» (49v); «... que linda... azia la parte de arriba con... el camino de las duernas... y al poniente con el camino de tras las casas que ba para Mansilla» (54v).

Camino Francés, El

«... a do llaman el camino franzes...» (68r).

\section{Camino Real, El}

Como el camino más importante del lugar, aparece nombrado numerosas veces, como límite de distintas tierras: «... que linda ... al poniente con el camino Real» (20v); «... que linda azia la parte derriba con el Camino Real» (66v). De manera semejante (es decir, como lindero) aparece en las páginas 20r, 55r,70v, 77r. En cambio, en 76v figura «... a do llaman el camino Real ...».

El Camino Real debió de ser el camino por excelencia y, con toda probabilidad, fue la referencia de otros topónimos como Allende el Camino. En la actualidad, el topónimo sigue vivo, aunque El Camino Real no coincide ya con la vía de acceso al pueblo.

Camino de Santovenia, El

«... a do llaman el camino de Santobenia ... y ba para Mansilla» (50v).

Camino de Tras las Casas, El

«.. que linda... azia la parte de arriba con... el camino de las duernas... y al poniente con el camino de tras las casas que ba para Mansilla» (54v).

Camino de Valdevela, El

«... a do llaman las questas de villa Sabariego ... que linda azia la parte de abajo con el camino de baldevela» $(52 \mathrm{v}) ; \ll \ldots$ que linda... azia la parte del oriente con el camino debaldebela» (53r).

Cárcava, La

«... a do llaman los zentenales ... y la atraviesa por el medio la 
carcaba»; «... a do llaman los zentenales de la carcaba ... que linda... y arriba con tierra del dean de león...» (57r). En la página $35 \mathrm{v}$, se dice que una tierra situada «a do llaman los callejos» no se siembra por ser un gran carcabal, es decir, se utiliza un abundancial de cárcava, para definir la calidad pésima de una tierra. Hoy en día permanece el topónimo La Cárcava.

Carrera de los Barriales, La

«... y azia el oriente con el camino que llaman de la carrera de los barriales» $(51 \mathrm{v})$.

Carros, Los

«... a do llaman los carros ... que linda con el camino que ba para nogales y con el camino que ba para Marne» (55v).

Centenales, Los

«... a do llaman los zentenales...» (48r, 49r, 49v, 55r, 55v; 56v, 57r, $65 \mathrm{r}, 84 \mathrm{r}, 105 \mathrm{v})$.

El topónimo Los Centenales sigue vivo probablemente referido al mismo lugar documentado. Pero también hemos recogido $E l$ Centenalón que ninguno de nuestros informantes identifica con alguno de los centenales documentados a continuación. A las tierras sembradas de centeno se les llama en Asturias centenal o centenel (DALLA, DGLA) y no es difícil encontrar allí topónimos semejantes como El Centenal, Centiales o Las Centiniegas, con sufijos que atestiguan una excelente fructificación del cereal (García Arias 2005).

Centenales de la Cárcava, Los

«... a do llaman los zentenales de la carcaba ... que linda... y arriba con tierra del dean de león...» (57r).

Centenales del Palomar, Los

«... a do llaman los zentenales del palomar...» (48r).

Centenales del Pradillo, Los

«... a do llaman los zentenales del pradillo...»(105r).

Cogullares, Los

«... a do llaman los cogullares... que linda... y azia abajo con tierra que llaman de la bariona» $(23 \mathrm{v}, 50 \mathrm{r}) ;$ «... a do llaman los Cogullares...» (82v, 83v).

Condado, El

«... a do llaman el condado linda... y al poniente con senda que sale al camino que es raya de villa verde y este lugar» $(15 \mathrm{r}, 52 \mathrm{r}, 63 \mathrm{v}, 79 \mathrm{v})$.

En 63v se habla de una «tierra linar», luego debe ser terreno de buena calidad. No siempre los topónimos que incluyen esa voz tienen que ver 
con antiguos condados. García Arias (2005) defiende que para explicar los topónimos asturianos El Condáu (Llaviana) y El Condao (Uviéu) habría que acudir a un nombre céltico CONDATE, 'confluencia de aguas', latinizado en CONDATUM. Al ser uno de los topónimos que ya no conocen en Mansilla Mayor, no podemos aportar datos en este sentido.

\section{Crucijadas, Las}

«... a do llaman las cruzijadas ... que linda... azia las partes del oriente y arriba con la rraya de mansilla Mayor y villaverde y lindera de conzejo» (61r); «... a do llaman las cruzijadas de los caminos ...» (61r); «... a do llaman la cruzijada de los caminos...» (70v).

La Cruciada o La Encruciyá (García Arias 2005) son dos topónimos asturianos que aluden a verdaderas encrucijadas o encruciyada, 'sitiu onde se crucien dos o más caminos, dos o más carreteres' (DALLA).

Cuestas de Villasabariego, Las

«... a do llaman las questas de villa Sabariego ... que linda azia la parte de abajo con el camino de baldevela» (52v).

Es un topónimo conocido hoy y muy usado.

Cueto Alto, El

«... que linda... azia la parte de arriba con tierra de santa Catalina de Santobenia y con el queto alto» (56r).

Cuetu o sus variantes cueto, сиӧtu se recogen en el DGLA con el significado de 'cresta', 'montículo', 'altozano', 'cerro', 'colina'. Son numerosos los topónimos asturianos recogidos por García Arias (2005) que llevan esta voz, como Cuetu, El Cuetu, El Cueto, Los Cuetos, El Cutulapila, Cutuperal, Cotillanu...

Deán, El

En la actualidad existe un lugar en Mansilla Mayor conocido como $E l$ Deán, pero la profusión de citas que aparecen en el Libro de Apeos hace pensar no tanto en un topónimo consolidado en aquel momento sino en referencias al propietario de diversas tierras cuando se lee $« . .$. linda... azía la parte de arriba con tierra del señor $d e A n$ de la ciudad de Leon»; «... con tierra de dehan de la ciudad de leon». Así aparece en $16 \mathrm{r}, 18 \mathrm{v}, 21 \mathrm{v}, 43 \mathrm{r}, 44 \mathrm{r}, 50 \mathrm{r}, 53 \mathrm{v}, 57 \mathrm{r}, 59 \mathrm{r}, 59 \mathrm{v}, 64 \mathrm{r}, 64 \mathrm{v}, 65 \mathrm{v}, 70 \mathrm{v}, 105 \mathrm{r}$. De todas las que, en aquel momento, se conocían como propiedad de esa figura eclesiástica, una de ellas ha quedado fijada como topónimo. Deán aparece en el DALLA como 'persona que tien un cargu na catedral inmediatamente inferior al del obispu'.

Duernas, Las

«... otra tierra que esta en el termino deste otro lugar a do llaman las duernas que aze una fanega de de senbradura trigal que linda...» (19r). 
Según García Arias (2005), duernu o duerna, 'artesa' se mantiene con vitalidad en asturiano y ha podido aplicarse a terrenos más o menos envallados o aptos para recibir aguas. De manera que ese apelativo daría nombre a lugares asturianos como El Duernu, La Duerna, Los Duernos, Les Duernes...

\section{Entremolino}

«... a do llaman entre molino...» (99v); «... a do llaman en tremolino...» (110r).

En la actualidad se sigue hablando de Entremolino y El Camino d'Entremolino, pero también hemos oído pronunciar Tremolino.

Entremolinos y corros

«... a do llaman entre molinos y corros...» (47r).

Era, $L a$

«... otra tierra que esta en el termino de santo benia y mansilla Mayor a do llaman la era ... que linda... y al poniente con el camino de la era» (54r).

En la actualidad hay varias eras: La Era l'Abadesa, La Era Flórez y Las Eras. Un estudio pormenorizado de sus límites permitiría identificar alguna de ellas con las que aparecen documentadas. El DGLA recoge variantes como eira, yera, aira.

Era de Arriba, La

«... a do llaman la fuente de la llama que aze tres carros de yerba... que linda ... azia las partes de abajo y arriba con campos de conzejo y con el canpo que llaman de la era de arriba» (24v); «... y azia la parte de arriba con la era que llaman de arriba» (51v).

Era del Barrio, La

«... otra tierra que esta en otro termino a do llaman el pradillo de las regantinas que aze tres eminas de senbradura trigal que linda azia la parte de arriba con tierra del convento de Sandobal que llaman la fontanilla... y azia la parte del poniente con la hera del barrio y madriz que tiene en medio.» $(15 \mathrm{v}) ;$ «... que linda azia el oriente con tierras digo con el camino de la hera del varrio.» (16v); «... a do llaman la era del barrio...» (89r, 92v y 113r); «... a do llaman la hera del barrio...» (92v).

Actualmente sigue existiendo La Era el Barrio.

Espino, El

«... a do llaman la vega de Marne... y sale aziendo pico a do llaman el espino» (45v).

En la actualidad existen Las Espineras, pero está muy lejos de La Vega Marne, referencia que aporta el Libro de Apeos, por lo que se trata de 
lugares distintos.

Hay un sinfín de núcleos de población en Asturias cuyo nombre incluye la voz espín o espinu, tanto en masculino, femenino, singular y plural (L'Espin, L'Espinu, Los Espinos, Espines...), como con diminutivos en -ĔLLAM (Espiniella, La Espinella ...) o con sufijo abundancial (Espinéu, Espinosa, Espinaréu...) (García Arias 2005).

Fontanal, El

«... a do llaman el fontanal a la era del barrio...» (113r).

Fontanilla, La

«... con tierra del convento de Sandobal que llaman la fontanilla... y azia la parte del poniente con la hera del barrio y madriz que tiene en medio.» (15v); en términos parecidos, es decir, una tierra propiedad del convento de Villaverde de Sandoval, se cita en 61v, 68r, 68v, 69r y $105 \mathrm{v}$.

En Asturias, Fontaniella (García Arias 2005).

Fragua, La

«... como llevador que es de un olmar que esta en el termino deste otro lugar a do llaman la fragua ...» $(77 \mathrm{v}) ;$ «... esta fundada sobre una Guerta que esta en el termino de mansilla mayo que llaman la martina a la calle de la fragua...» (108v).

En Mansilla Mayor recuerdan todavía fraguas de distintos herreros de finales del siglo XIX y también del XX (Villafañe Llorente 2018: 182) pero debió de haber una fragua que ya en el año de este Libro de Apeos pasó de ser un edificio a un prado, dado que en la página 109v se dice: «... un Prado que antes fue casa que esta en el termino deste otro lugar de Mansilla Mayor a do llaman la fragua... que linda azia la parte de abajo con casa y Guerto de Joseph Llorente y al poniente con Prado y olmar de Gregorio dela Presa y casa del otro llevador y al oriente y arriba con calles de conzejo». Este mismo desplazamiento semántico lo refleja García Arias (2005) para los topónimos La Fragua o Las Frauguas.

Fuegos, Los

«... al poniente con el camino que biene de la carrera que ba para los fuegos y con lindera de conzejo» $(18 \mathrm{v}) ;$ «... otra tierra que esta en el termino deste otro lugar a do llaman los fuegos que aze tres eminas de desenbradura trigal que linda...» (19r).

De manera parecida a las anteriores se cita en $19 \mathrm{v}, 48 \mathrm{v}, 60 \mathrm{r}, 83 \mathrm{v}$ y $81 \mathrm{r}$ (en este caso con mayúscula, los Fuegos). Creemos que las referencias de las páginas $24 \mathrm{r}, 69 \mathrm{v}$, donde se lee Juegos, son variantes del mismo topónimo. Hoy en día sigue existiendo un lugar conocido como Los Fuegos. 
Fuente de Arriba, La

«... a do llaman la fuente de arriba...» (91v, 92r), debía de ser una de las fuentes situadas en el casco del pueblo por las referencias a casas y huertas del mismo.

Fuente del Cuervo, La

«... a do llaman la fuente del cuerbo... que linda azia la parte del oriente con la rraya que dividen los términos de mansilla Mayor y villa Sabariego... y abajo con linderos y juncal de conzejo» (56v), aunque no se aclara si es de Mansilla Mayor o de Villasabariego.

Fuente de la Llama, La

«... a do llaman la fuente de la llama que aze tres carros de yerba...» $(24 \mathrm{v}) ; \ll \ldots$ el rreguero de la fuente de la llama ... y otro Prado esta en el termino deste otro lugar a do llaman la fuente dela llama» (74r).

En la actualidad, sigue existiendo una fuente conocida como La Fuente la Llama.

Fuente de Santovenia, La

«... otra tierra que esta en el termino de Santobenia a do llaman la fuente de santobenia que aze dos eminas de senbradura trigal que linda... azia la parte de arriba con camino detrás las casas de otro lugar y azia el poniente con canpo de conzejo.» (17v).

Gollón, El

«... a do llaman el gollon ...» (80r).

En Asturias un gollón es un 'valle estrecho y pequeño, vaguada' (DGLA), 'valle pequeñu' y 'parte más fonda d'un valle, d'una finca' (DALLA). Al no ser un topónimo conocido en Mansilla Mayor no podemos aportar datos que confirmen esa descripción.

Granja de la Mansilleja, La

«... a do llaman la granja de la mansilleja...» (91r).

Grulleras, Las

«... otra tierra que esta en otro termino de Mansilla Mayor a do llaman las Grulleras que aze dos eminas... que linda... y azia la parte de abajo con el que llaman de los lombos...» (15r).

Huelga, La

En el margen de la p. $16 \mathrm{v}$, pone «Otra a la guelga», pero en el deslinde que se hace no se nombra.

Huertas de Arriba, Las

«... a do llaman la guerta de arriba ...» (85r); «... a do llaman las guertas de Arriba...» (103r); «... a do llaman las Guertas de arriba...» $(105 \mathrm{v})$. 
Huertas de la Malatería, Las

(Ver La Maletería)

Laguna Salgada

«... a do llaman Laguna Salgada que aze de senbradura dos eminas trigal que linda azia las partes del oriente y arriba con tierras de las Monjas de gradefes y abajo con lindero de conzejo y al poniente con lindero de conzejo y la atraviesa la madriz grande de conzejo» (23r).

La palabra asturiana salgada, 'planta rastrera que se cría en los sembrados' (DGLA) tiene presencia toponímica también en Asturias en La Pena la Salgada, según García Arias (2005).

Lagunas, Las

«... otra tierra que esta en el termino de otro lugar de Mansilla Mayor a do llaman las lagunas...» (18r, 24r, 50r, 51r, 64v, 112v).

Además de aparecer documentado varias veces, se alude a su carácter «linar», esto es tierras de buena calidad. Hoy sigue existiendo el topónimo pero, por efectos de la concentración parcelaria, ya no existen las lagunas que originaron el nombre del lugar. En Asturias hay numerosos topónimos procedentes de LACUM y de LACUNAM cuya forma presenta la palatalización de L- (García Arias 2005).

Lavaderos, Los

«... a do llaman a los labaderos... que linda... azia el oriente con la presa del conzejo y azia el poniente con lindero de conzejo» (46v, 47r).

Lombos, Los

«... que llaman de los lombos...» (15v).

El topónimo permanece hoy con la misma forma. Llombu o l.lombu y sus variantes femeninas llomba o l.lomba han formado topónimos asturianos como La Llomba, la L.lomba, Lomba, Les Llombes o El Llombón (García Arias 2005).

\section{Llama, La}

«... a do llaman la llama... que linda azia el poniente con el reguero de la fuente de la llama...» (88r). También aparece citado en $73 \mathrm{r}$ y en $112 \mathrm{r}$ se habla de «la guerta de la llama», de la que se dice que «... linda con otra casa y calles de conzejo...», es decir, estaba en el casco del pueblo, hecho que también atestigua la referencia a «el barrio de la fuente de la llama» (111r).

En la actualidad siguen existiendo La Llama, un lugar que, efectivamente, se sitúa pegado al casco del pueblo. Topónimos muy frecuentes en territorio asturiano son La Llama, La L.lama, Llames, Llamas, con sufijo diminutivo La Llamiella, o abundativo La Llamosa (García Arias 2005). 
Madrices, Las

«... a do llaman las madrizes ... que linda... al oriente con las madrizes de conzejo y con el camino que ba a villa Sabariego» (58r) y de forma semejante se recoge en $52 \mathrm{v}, 58 \mathrm{v}, 66 \mathrm{r}, 66 \mathrm{v}$ y $67 \mathrm{r}$.

En $70 \mathrm{v}$ se puede leer de un lugar $« \ldots$ a do llaman las madrizes de Pradillo...» que hoy en día nadie reconoce, pero sí sigue existiendo un topónimo Las Madrices.

Maletería, $L a$

«... a do llaman lámala/teria ... y azia abajo con el reguero del caño de la malateria» (76v). En 67v se habla de un lugar «... a do llaman tras las guertas del caño de la melateria ... que linda azia la parte del oriente con tierra de la eredad del dean de león... y azia abajo con el rreguero de la malatería...».

Actualmente, La Maletería se identifica claramente. Igual que en León, los topónimos como La Maleta, La Malatería, la Maletería (García Arias 2005) atestiguan en Asturias la existencia de antiguas malaterías $\mathrm{u}$ hospitales de malatos o leprosos.

Mangas, Las

«... otra tierra... que esta en el termino deste otro lugar a do llaman las mangas que aze dos eminas de senbradura trigal que linda azia la parte de abajo y oriente con el camino que ba a mataganado y villa sabariego ... y arriba con el camino Real.» (20r) y de manera parecida en 20v y 65v.

García Arias (2005) relaciona el topónimo asturiano El Mangueiru con la misma base latina MANICAM, origen de manga, voz que, en Asturias, alude a una franja de terreno un tanto orillada.

Mansilleja, La

«... a do llaman la mansilleja ... que linda ... azia arriba con tierra de froilan romero apeador y aze pico azia mansilla y al oriente con la rraya» (24r); «... a do llaman la granja de la mansilleja...» (91r).

Es un topónimo que sigue existiendo. Algún informante incluso habla de Las Linares de la Mansilleja.

Manteca, La

En el margen de 16r, pone «Otra a la manteca» aunque luego no se nombra cuando enumera los límites; en $80 \mathrm{r}$ sí se lee, «... a do llaman $l a$ manteca...».

Pervive en la actualidad como La Manteca.

Martina, La

«... esta fundada sobre una Guerta que esta en el termino de mansilla mayo que llaman lamar tina a la calle de la fragua... que linda... y al poniente con Prado y olmar de Gregorio...» (108v). 


\section{Mataganao}

«... a do llaman mataganado ... que linda... arriba con tierra de la fontanilla. » (61v) y de manera semejante en $67 \mathrm{v}$. El topónimo aparece citado bastantes más veces pero para indicar límites de otras tierras o prados. Por ejemplo: «... que linda azia la parte de abajo y oriente con el camino que ba a mataganado y villa sabariego» (20r y, de manera semejante en 19v, 20v, 48r,60r, 81r).

Pervive en la actualidad como Mataganao.

\section{Media Villa, La}

El lugar «... a do llaman la media villa...» se cita en 43r; 44r, 63v, 95v. Aparece cuando se habla de propiedades que estaban en el propio casco del pueblo, como ilustra esta cita: «... y otros encargos están fundados sobre un erreñal que esta en el casco de este otro lugar de Mansilla Mayor a do llaman la media villa...» (88v).

Molino, El

«... a do llaman el molino...» (43r).

Todavía existe El Molino (topónimo y edificio). La toponimia asturiana utiliza la voz molín (García Arias 2005).

Nogal, El

En 47r, pone al margen nogal, aunque luego no se cita en el deslinde que se hace de la tierra, un lugar que lindaba «... con el camino que ba de el lugar de santo benia para el molino». En $82 \mathrm{v}$ aparece «... a do llaman los nogales...», probablemente dos lugares diferentes; no obstante, la información aportada por el Libro de Apeos no nos permite inferir que se trata del mismo lugar ni que se trata de dos lugares diferentes.

Hoy no dan cuenta de ninguno de los dos topónimos (aunque hay un pueblo muy pequeño colindante que se llama Nogales). Aunque García Arias (2005) recoge un Negales, en la toponimia asturiana son mucho más frecuentes los topónimos tipo Nocéu, La Nozal, Nozalín... o los colectivos Noceda, La Nozaleda, La Nozalea (que contienen el nombre del árbol que da nueces) que los de tipo nogal.

Paredicas, Las

«... a do llaman las paredicas ...» $(23 \mathrm{r}, 82 \mathrm{v})$.

\section{Ponjal, El}

«... a do llaman el berjel que esta en la media villa ... que linda... y azia la parte de abajo con Prado que llaman el ponjal» (63v).

Las indicaciones del Libro de Apeos nos hacen identificarlo con lo que hoy conocen como El Ponjalete.

Pontonzilla, $\mathrm{La}$

$\ll \ldots$ y rreguero que ba a la pontonzilla» $(44 \mathrm{r}, 77 \mathrm{r})$. 
Topónimos como El Pontón, Pontón o El Pontón de Vaqueros, que recoge García Arias (2005) incorporan el sufijo aumentativo, aunque el que aparece en nuestro Libro de Apeos viene doblemente sufijado, primero con un aumentativo y luego un diminutivo (algo aparentemente contradictorio).

Poza, La

«... a do llaman la poza ...» (25v, 80v).

La voz poza es conocida en asturiano (DALLA y DGLA), pero los nombres de lugar que aporta García Arias (2005) aparecen todos en masculino.

Pradillo, El

«... a do llaman el Pradillo...» (25r; 53v, 80r). En 15v se habla de «... otra tierra que esta en otro termino a do llaman el pradillo de las regantinas...», aunque no podemos saber si se trata de dos lugares diferentes.

Prado de los Barreros, El

«... a do llaman el Prado de los barreros...» (94r), que hoy conocen como Los Praos Barreros.

El apelativo barreru, $-a$, - $o$, 'con barro' (DALLA), 'barroso' (DGLA) aparece en numerosos topónimos asturianos que García Arias (2005) califica de abundativos: Barréu, Barredo, El Barreiru, La Barrera, Los Barreos, Barreres...

Prado de Fuzia Núñez, El

«... a do llaman el Prado de Fuzia nuñez...» (44r).

Prado del Palomar, El

«... que llaman el Prado del palomar ...»(63r).

Debía de ser un prado que estaba en el casco del pueblo porque lo citan para limitar la casa de un vecino, Gregorio de la Presa. Los topónimos asturianos que se forman con la voz palombu, palomba conservan también el grupo -MB- (cosa que no ocurre con este topónimo de Mansilla Mayor): El Palombo, La Palomba, Les Palombres, Palombar, La Palombera... (García Arias 2005).

Prados de Arriba, Los

«... a do llaman los Prados de arriba ...» (72r).

Prados del Puerto Abertizo, Los

«... a do llaman los Prados del puerto abertizo... que linda ... azia la parte de arriba con rreguero de la fuente de la llama...» (44r).

Prados de la Vega, Los

«... a do llaman los Prados de la vega ...» (114r).

Puerto, El

«... a do llaman el Puerto ...» $(24 \mathrm{v}, 25 \mathrm{r})$.

Es posible que se corresponda con el actual El Portico. No creemos que 
este topónimo se pueda relacionar con los recogidos por García Arias (2005) (El Puertu, Puerto, Porto, El Porto, Puertu Veiga) y que se refieren a lugares de paso en la montaña o a sitios abrigados en la costa donde se anclan las embarcaciones.

Ramal, El

«... a do llaman el ramal» (43v, 44r, 54r, 113v).

Pervive hoy con la misma forma.

Raposa, La

«... a do llaman la rraposa ...» $(15 \mathrm{v}, 79 \mathrm{v})$.

Actualmente, Las Raposeras. Los topónimos asturianos La Raposa, El

Raposu son testimonios de la palabra asturiana con la que se designa a la zorra (García Arias 2005).

Regantina, La

«... otra tierra que esta en otro termino a do llaman la regantina » $(16 \mathrm{v}$, $105 v)$. Y en $15 \mathrm{v}$ se habla de $« . .$. otra tierra que esta en otro termino a do llaman el pradillo de las regantinas...» cuyo deslinde permite situarlo en la misma zona.

Reguero de la Fuente de la Llama, El

«... a do llaman los Prados del puerto abertizo... que linda ... azia la parte de arriba con rreguero de la fuente de la llama» (44r, 74r, 88r).

Los apelativos asturianos regueru, reguera, regatu, riegu están presentes (con diferentes variantes) en numerosos nombres de lugar de Asturias, tal como recoge García Arias (2005).

Reguero de la Malatería, El

(Ver La Maletería).

Reguero del Caño de la Malatería, El

(Ver La Maletería).

Requejada, La

«... a do llaman la rrequejada ... que linda... azia arriba ... y con la rraya que dibide los términos de Mansilla Mayor y Villa moros...» (65r); asimismo se cita en 47r y 83 r.

Topónimo que pervive con la misma forma, La Requejada. Con las variaciones fonéticas propias del asturiano, aparecen topónimos como La Requexada, La Requeixada (García Arias 2005) semejantes a este de Mansilla Mayor.

Salida para Nogales, La

«... a do llaman la salida para nogales...» (93v).

Santa Catalina

«que linda... con camino que ba a do llaman Santa Cathalina...» (49r, 
56v); «... a do llaman Santa Catalina... que linda... azia la parte de arriba con tierra de santa Catalina de Santobenia y con el queto alto» (56r).

También en Asturias aparece el nombre de esta santa en la formación de algún topónimo (García Arias 2005).

Sobrelera

«... a do llaman sobre laera ... que aze dos carros de yerba...» (25r);

«... a do llaman Sobrelera...» (103v).

El topónimo actual es Sobrelera.

Sollanzo

«... otra tierra que esta en el termino deste otro lugar a do llaman Sollanzo...» (18v, 52v, 59v, 64r, 68r, 68v, 69r, 77r).

Topónimo muy interesante, que se mantiene en la actualidad con la misma forma, pero con peligro de desaparición porque se ha trazado por allí una autovía inaugurada en 2018.

Tiñosas, Las

«... a do llaman las tiñosas...» $(21 \mathrm{v}, 60 \mathrm{v})$.

Titales, Los

«... otra tierra que esta en otro termino de Mansilla Mayor a do llaman los titales qu eaze media carga de senbradura trigal...» (18r; 18v, 23r). Pervive en la actualidad con la misma forma: Los Titales.

Tras las casas

«... una tierra que esta en el termino del lugar de Santobenia a do llaman tras las casas...» (15r); «... una tierra que esta en el termino de Santobenia tras las casas... y al poniente con el camino de tras las casas que ba para Mansilla» (54v); «... que linda... azia la parte de arriba con camino de trás las casas de otro lugar y azia el poniente con canpo de conzejo» $(17 \mathrm{v})$.

Tunba, La

«... a do llaman la tunba ... y la atraviesa por medio la madriz grande» $(60 \mathrm{v}, 105 \mathrm{r})$.

Quizá haya que relacionar este topónimo con El Tombín o El Tombo estudiados por García Arias (2005).

Vadua, La

«... a do llaman la badua ... que linda... al poniente con pradera de conzejo» $(52 \mathrm{v})$.

En la actualidad existe el topónimo Las Badugas, topónimo semejante al que García Arias (2005) recoge como La Vaduga (junto con otros varios topónimos asturianos del tipo La Vaúa, La La Vaúba, La Vayuga...) con un posible origen el el latín VADUM, castellano vado y asturiano vau. 
Vago, $E l$

«... otra tierra que esta en otro termino a dizen bago que aze cuatro eminas zentenal de senbradura que linda azia el oriente con lindera de conzejo... y azia la parte de abajo con camino que ba para Mansilla y para Sandobal» (17r).

Vagu es palabra bien conocida en asturiano con el significado de 'ería, conxuntu [de tierres xuntes]' (DALLA).

Vega, La

«... una tierra que esta en termino de este otro lugar a do llaman la vega que aze ocho eminas de senbradura trigal» $(17 \mathrm{v})$.

En toponimia asturiana, el apelativo veiga o vega aparece en numerosísimos topónimos, bien en expresiones simples (Vega, Las Veigas, Les Vegues...), compuestas (Vegamaor, Veigafriosa, Veigalladrona, La Veiga'l Castru, La Vega Riba... ), derivados (La Vegona, La Veiguiella... ) (García Arias 2005).

Vega Marne, La

«... a do llaman la vega de Marne...» (45v, 46r, 83r).

Hoy en día se conoce como La Vega Marne.

Velilla, La

«Otra a la belilla» (16v).

En la actualidad, La Velilla, cuyo correlato en Asturias podría ser $L a$ Viliella (García Arias 2005).

Vergel, El

«... a do llaman el berjel que esta en la media villa...» (63v, 106v); «... y esta fundado sobre un Prado ponjal que esta en el termino de este otro lugar a do lla / los berjeles... que linda azia la parte de arriba con el Prado que llaman el berjel y al poniente y abajo con calles de conzejo» $(106 \mathrm{v})$.

\section{Topónimos documentados en el Libro de Apeos de MANSILla MAYOR QUE PERTENECEN A OTROS PUEBLOS CERCANOS}

Tanto la iglesia de Mansilla Mayor como los habitantes del pueblo tenían propiedades no solo en el territorio de este pueblo sino también en el de otras poblaciones cercanas y así se refleja en el Libro de Apeos, Aniversarios, Fundaciones, Cofradías de Mansilla Mayor. Como no hemos recogido la toponimia de esos lugares (al contrario de lo que sucede con la de Mansilla Mayor), no podemos saber si esos topónimos documentados son conocidos todavía hoy o ya se han perdido. 
En la localidad de Marne:

Soto de Marne, El: «... a do llaman el soto de Marne...» (46r).

Vallina, La: «... a do llaman la ballina ...» (46v).

Vega, La: «... que esta en el termino de el lugar de Marne a do llaman la bega ...» $(57 \mathrm{v}, 104 \mathrm{r})$.

En la localidad de Nogales:

Portico, El: «... en el termino de nogales a do llaman el portico...» (45r y $45 \mathrm{v})$.

En la localidad de Valle de Mansilla:

Entre las páginas 27 a 41 se recogen las numerosas propiedades que la iglesia de Mansilla Mayor tenía en Valle de Mansilla, población situada a menos de $10 \mathrm{~km}$. Los topónimos de esta localidad que se citan son:

Los Arenales (28v).

Los Callejos (35v).

El Camino de Cañones (36v).

El Caño: «... a do llaman el caño... que linda... y azia arriba con la questa que llaman del caño...» (37r).

La Carbacera: «... a do llaman la carbazera...» (27v).

La Cárcaba (35r).

Carre Mansilla (32r).

Carro Sorriba (31v).

Los Cenizales: «... a do llaman los zenizales...» (35r).

El Cercado: «... a do llaman el zencado...» (40v). No obstante, en el margen pone «cercado».

Las Cuevas y La Cuesta las Cuebas: «... a do llaman las cuebas... que linda... y azia arriba con la questa de las Cuebas...» (37r).

La Cuesta de los Higales: «... a do llaman el socastro... que linda... y azia arriba con la questa de los Ygales...» (35r).

La Cuesta del Horno, cuya transcripción varía entre questa del horno $(34 \mathrm{v})$ y la questa del orno (40v).

La Cuesta de Solorno (escrita como questa de solorno, 36r).

Entrepresas: «... a do llaman entre presas... que linda ... azia arriba con la presa del casar...» (41r).

El Escobar (30v, 31r) y El Camino del Escobar (31r).

El Espino (31r).

La Huerta, La Huerta de Abajo: «... a do llaman la Guerta... linda... con el camino de la Guerta de abajo » (27v, 29 r).

Mangas (39v).

El Molino Caído (34r, 38v), El Camino del Molino Caído (40r) y Las Vallinas al molino Caído (34r). 
El Molino del Medio (29r).

La Muela $(28 \mathrm{v}, 33 \mathrm{r})$.

Palacio (27v, escrito palazio), Los Palacios (28r, escrito palazios).

El Pico (27r).

El Picón (28v).

El Pontón de Piedra (40v).

El Portezuelo de las Vallinas (34r).

La Presa del Casar (36r, 36v, 37v, 38r, 40v, 41r).

La Presa del Soto (ver El Soto).

La Presa de Valdeloria (ver Valdoria).

El Reguero de la Jadria (30v) o El Reguero de la Judria (32r).

El Recorbo: «... a do llaman el rrecorbo...» (39r).

Los Renajales: «... a do llaman a los renajales...» (31r).

El Ronzal (34v) y El Camino del Ronzal (38v).

El Saugo (y al margen con la forma Sabugo) (38r).

El Sendero (31v, 41r).

Las Sernas (37v, 39v) y Las Sernas de Abajo (39v).

Sillanueva: $« \ldots$ a do llaman a silla Nueba $=$ digo Socastro $=\ldots »($ en el margen pone «Sillanueba digo Socastro») (36v).

Socastro (34v, 35r, 36v, 40v).

Solorno (36r, 36v).

El Soto (39v, 40r), Los Sotos (30r), junto con La Manga del Soto (29v, $38 \mathrm{r})$ y La Presa del Soto $(38 \mathrm{v}, 39 \mathrm{v})$.

El Tejar (28r).

La Tierra de Inés: «... a do llaman la tierra de Ynes...» (32r); «... a do llaman tierras de ynes...» (30v).

Traslaviña: en el margen se puede leer viña y, luego, en el deslinde «... a do llaman traslabiña...» (35v).

Valdoria. Un topónimo frecuentemente citado como baldoria (40r), junto con «... la presa de conzejo que llaman de baldeloria» (39r, 34 v), la presa de la baldiloria (38v) y los tesos de baldeloria (40r).

Las Vallinas, aparece indistintamente con «V» (las vallinas, 29v, 30r, $33 \mathrm{v}, 38 \mathrm{r}$ ) o con «b» (ballinas, 33v, 38r; las ballinas al molino caído, $34 \mathrm{r}$ ).

Las Zapatas (38v).

En la localidad de Villacelama:

La Gatona, La Gatona de Piedra (44v y 45r).

En la localidad de Villamoros:

El Fontanal (52r).

En la localidad de Villasabariego:

La Cárcaba (53v) 
El Castro «... a do llaman el castro... que linda azia la parte de arriba con el camino de la carrera y al oriente con lindero de conzejo que tiene una poza» (56r). El topónimo sigue vivo hoy: es la única elevación del terreno desde donde se divisa todo el término de Mansilla Mayor.

La Cuesta el Castro «... a do llaman la cuesta del castro» (56r). Como El Castro, La Cuesta el Castro es un topónimo bien conocido hoy por los habitantes de Mansilla Mayor.

Cueto de Valdelaguna, transcrito como cueto de baldelaguna (53v).

Tras Fosorio [en margen, fosorio] (53v).

Valdecontintrigal (62r)

Valdevela y El Camino Valdevela: «... en el termino de otro lugar de billasabariego a do llaman baldebela ... que linda... azia la parte del oriente con el camino de baldebela» (53r). En 52v se vuelve a hablar de El Camino Valdevela: «... a do llaman las questas de villa Sabariego ... que linda azia la parte de abajo con el camino de baldevela».

\section{CONCLUSIONES}

En la presente investigación se ha procedido a la recuperación de cada uno de los topónimos contenidos en el Libro de Apeos de Mansilla Mayor y la primera de las conclusiones que destacaríamos tiene que ver con el número de topónimos que ya no se conocen en la actualidad y que sí aparecen en ese documento de 1746: de los 108 topónimos documentados para el término de Mansilla Mayor, actualmente solo perviven 33, es decir, un 30,5\%. Podríamos elevar un poco este porcentaje porque hay lugares que se reflejan de manera muy genérica (como eras o caminos), que actualmente siguen existiendo. Por ejemplo: en lugar de La Era o La Era de Arriba (citadas respectivamente en 54r y 24v) hoy hablan de La Era l'Abadesa, La Era Flórez o Las Eras, un terreno comunal donde cada vecino tenía un trozo asignado para depositar allí la mies segada con el fin de trillarla, limpiarla para separar el grano de la paja y poder almacenar así los productos obtenidos. En el caso de los caminos, la concentración parcelaria ha hecho que el trazado de estas vías de comunicación cambie completamente. El dato, la pérdida de un 68 o $70 \%$ de los topónimos documentados en el Libro de Apeos, es muestra de la necesidad de recoger esos nombres de lugar.

Hemos considerado que los topónimos citados como El Ponjal (63v) o El Puerto $(24 \mathrm{v}$ y $25 \mathrm{r})$ se corresponden con los actuales El Ponjalete y El Portico, aunque habría que estudiar si los límites citados en el Libro de Apeos los sitúan en zonas donde hoy nos dicen que están. Dos casos curiosos son $E l$ Deán, profusamente citado y referido a posesiones en distintos puntos de 
Mansilla Mayor, aunque hoy solo identifican un lugar llamado así y Las Lagunas, topónimo conocido y perfectamente identificable, pero con muchas posibilidades de desaparición porque, también por efecto de la concentración parcelaria, han desaparecido las lagunas que motivaban su nombre.

Otras formas toponímicas que destacaríamos por ser tradicionales son El Castro, por el valor arqueológico que tiene al señalar un asentamiento primitivo; Las Crucijadas, por la costumbre de señalar los cruces de caminos; o La Llama, un topónimo de abundante presencia en el Noroeste peninsular. Habituales también son los topónimos relativos, esto es, aquellos cuyo nombre lo sitúa respecto a otro, de manera que son necesarios los dos nombres de lugar para quedar bien definidos, usando para ello preposiciones latinas como SUB (Sollanzo, Solorno, Socastro), SUPER (Sobrelera) o adverbios como Tras las Casas, Tras Fosorio.

Destacaremos dos fenómenos lingüísticos habituales en toponimia y que encontramos en algunos de los topónimos incluidos en este Libro de Apeos: uno es la presencia del sufijo abundancial (o colectivo) -al en Los Barriales, Los Centenales, El Ponjal, Los Cenizales, La Cuesta de los Higales, por ejemplo; otro es la conservación del grupo latino -MB- en Los Lombos, pero no así en El Prado del Palomar.

Otro grupo de conclusiones tiene que ver con el vocabulario. Desde el punto de vista lingüístico y dejando a un lado los topónimos, es interesante encontrar documentadas palabras cuyo uso hoy ha desparecido o está en franco retroceso. Así, la palabra ejido cuyo significado, según el DRAE, es «Campo común de un pueblo, lindante con él, que no se labra, y donde suelen reunirse los ganados o establecerse las eras», que hoy no conocen, aparece documentado dos veces como apelativo común para indicar linderos de algunas propiedades: «... que linda... al poniente y oriente con ejidos de conzejo». (40v); «... que linda de las demás partes ejidos de conzejo» (52r).

La voz ferreñal que aparece en Le Men (2005) con el significado de 'terreno en que se siembra el herrén', figura documentada en el LAA-1 con esa misma forma (ferreñal, 64a) y también como ferrenal (75a), erreñal (75a, 88 b) y su plural erreñales (75a, 107a). Se usan en el Libro de Apeos para indicar límites de propiedades que están en el casco del pueblo (en la media villa). Las formas documentadas en Mansilla Mayor recogen la vacilación entre ferreñal y la castellanizada herreñal que sí recoge el DRAE.

Otras dos palabras que rescatamos porque no las recoge el DRAE y cuyo uso en el pueblo es prácticamente nulo en la actualidad son olmar y ponjal, dos abundanciales fitotoponímicos, que señalan lugares donde abundan los olmos (o negrillos) y los ponjos (o chopos) respectivamente. En la página 77b del LAA-1 se cita a una persona «... como llevador que es de un olmar que esta en el termino...» y en la página $108 \mathrm{~b}$ se delimita una propiedad diciendo 
que linda «... al poniente con prado y olmar de Gregorio...». Olmar no es una voz que aparezca en el léxico del asturianoleonés actual, pero sí ponjal (Le Men 2005) que, aunque como desusado, significa 'zona plantada de chopos o álamos' y lo localiza en la comarca leonesa de Los Oteros (Morala 1990: 293), no muy alejada de Mansilla Mayor. En la página 106b de LAA-1 se habla de una propiedad «... y esta fundado sobre un Prado ponjal que esta en el termino de este otro lugar...». En Mansilla Mayor todavía hoy hemos recogido como topónimo vivo El Ponjalete, uno de los lugares donde mejor se daban los chopos.

Aunque no aparece usado como apelativo en el Libro de Apeos (lo que sí sucede con olmar o ponjal), el topónimo documentado en 80r, El Gollón, podría ser testigo de que la palabra asturiana gollón 'valle estrecho' que aparece tanto en el DGLA como en el DALLA se usaba otrora en este lugar.

Definitivamente, el hallazgo del Libro de Apeos, Aniversarios, Fundaciones, Cofradías de Mansilla Mayor nos ha permitido obtener datos toponímicos muy interesantes, y también algunos datos léxicos, de una zona que hoy en día sufre una despoblación considerable y cuya orografía ha sufrido un cambio drástico debido a la concentración parcelaria de hace treinta años y a la reciente construcción de una autovía.

\section{Bibliografía}

Corominas, J. (1972): Tópica Hespérica. Madrid, Gredos. (2 volúmenes).

DALla (2007): Diccionariu de la llingua asturiana. Uviéu, Academia de la Llingua Asturiana. [Consultado en http://www.academiadelallingua.com/diccionariu/index.php].

DGLA: García Arias, X. Ll. (2005): Diccionario General de la Lengua Asturiana. Oviedo, Prensa Asturiana. [Consultado en https://mas.lne.es/diccionario/].

García ArIAS, X. Ll. (1995): Toponimia: teoría y actuación. Uviéu, Academia de la Llingua Asturiana.

- (2005): Toponimia asturiana. Oviedo, Prensa Asturiana. [Consultado en https://mas.lne.es/toponimia/].

García-SAmpedro Clérigo, C. (2008): «El Apeo del «Concejo de Oviedo» en El Libro del Prior (I)», en Lletres Asturianes 99: 7-56.

GONZÁLEZ y FERnÁNDEZ-VALles, J. M. (1959): Toponimia de una parroquia asturiana. Oviedo, IDEA.

LAPESA, R. (1969): «La toponimia como herencia histórica y lingüística», en Las Ciencias XXXIV: 239-251.

Le Men, J. (2005): Léxico del leonés actual. León, Caja España de Inversiones \& Archivo Histórico Diocesano de León.

MenÉndez PidAl, R. (2018): El dialecto leonés. (Facsímil de la primera edición y encuestas, con textos actuales en leonés y audio). León, El Búho Viajero.

Miranda Pérez-SEOANe, J. (1985): Contribución al estudio de la toponimia menor de la cuenca alta del Esla (León). León, Diputación de León, Institución Fray Bernardino de Sahagún, (2 volúmenes).

Morala Rodríguez, J. R. (1984): La toponimia de una zona del Esla. Palanquinos, Campo y 
Villavidel. León, Universidad de León.

- (1990): Toponimia de la comarca de Los Oteros (León). León, Diputación Provincial de León. (2 volúmenes).

Ponce MolinA, P. (1977): «Fuentes para el estudio de la Geografía agraria de Andalucía Oriental: Los libros de Apeo y Repartimiento del último tercio del siglo XVI», en Medio físico, desarrollo regional y geografía: $V$ Coloquio de Geografía (3 al 6 de octubre de 1977). Granada, Facultad de Filosofía y Letras de la Universidad de Granada-Sección de Geografía: 289-296.

Real ACAdemia Española (2001): Diccionario de la lengua española. [Consultado en http://www.rae.es/rae.html (actualización de 2017)].

Villafañe LloRente, M. (coord.) (2018): Mansilla Mayor. Un recorrido por su memoria. León, Lobo Sapiens. 